\title{
Investigating the GALILEO and BeiDou orbit accuracy derived from rapid products
}

\author{
${ }^{1}$ Sermet Ogutcu, ${ }^{1}$ Salih Alcay and ${ }^{* 1}$ Omer Faruk Atiz \\ ${ }^{* 1}$ Engineering and Architecture Faculty, Geomatics Engineering Department Necmettin Erbakan University, Turkey
}

\begin{abstract}
In recent years, the advances of the new Global Navigation Satellite System (GNSS) constellations including, Galileo and BeiDou (BDS), have undergone dramatic changes. Some analysis centers (ACs) produce precise orbit and clock products of Galileo and BeiDou constellations. Currently, three types of Galileo and BeiDou satellite orbit and clock products are available - namely, precise, rapid and ultrarapid products -. Ultra-rapid and rapid products are generally used for time-constrained applications. Precise orbit determination (POD) of Galileo and BeiDou is much challenging compared with GPS and GLONASS constellations due to the officially undetermined receiver phase center offset (PCO), variations (PCV) of Galileo and BeiDou constellations and, also some other not well-defined factors such as yaw-attitude models and solar radiation pressure. In this study, GALILEO orbit accuracy is investigated using rapid products produced by Center for Orbit Determination in Europe (CODE) GeoForschungsZentrum (GFZ) and Wuhan University (WUHAN), while GFZ and WUHAN rapid products are used for BeiDou constellation only. One month (January) of data in 2020 is used to compute errors of radial, along-track, and cross-track components of Galileo and BeiDou orbit derived by rapid products compared with the CODE final Multi-GNSS Experiment (MGEX) product which is assumed as the reference product. The results show that no significant differences between the products are found for Galileo orbit. For BeiDou orbit, WUHAN rapid product produced the smaller root mean square errors (RMSEs) of orbit components compared with the GFZ rapid product.
\end{abstract}

Key words: BeiDou, Galileo, GNSS, Rapid Product

\section{Introduction}

Precise Global Navigation Satellite System (GNSS) applications require precise orbit and clock products generated by analysis centers (ACs) [1]. Five types of GNSS products are available namely, broadcast, ultra-rapid (predicted), ultra-rapid (observed), rapid and, final products - [2]. The broadcast ephemeris is mainly used for navigation applications that require meter or sub-meter level accuracies. Ultra-rapid predicted part is mainly used for real-time applications. Ultra-rapid observed part is generated for near-real time applications. The most accurate orbit and clock product (except for final product) for time-constrained applications is rapid product. The last product which is assumed the most accurate product is the final product [3]. Because of the users demand, ultra-rapid and rapid products have been generating with less delay compared with the final product for the time-constrained applications [4]. The latency and accuracy differences between the products depend on the processing strategy and number of used GNSS stations by ACs [5]. Generally, fewer stations are used for generating of ultra-rapid and rapid products compared

*Corresponding author: Address: ${ }^{1}$ Necmettin Erbakan University Engineering and Architecture Faculty, Geomatics Engineering Department, Turkey 42140, Konya TURKEY. E-mail address: oatiz@erbakan.edu.tr 
with the final product. As a result, the integrity and accuracy of ultra-rapid and rapid products are much less compared with the final product. The Multi-GNSS Experiment (MGEX) [6] project was initiated by the International GNSS Services (IGS) in 2011 to integrate the new emergence GNSS constellations, Galileo and BeiDou. Full operational capability (FOC) of Galileo has not been reached unlike that GPS and GLONASS. BeiDou constellation consisting of two parts - namely BeiDou-2 and BeiDou 3 -. BeiDou-2 satellites are mainly operating in Asia-Pacific region whereas, BeiDou 3 satellites are mainly operating globally. But due to the receiver and hardware issues, most of the GNSS stations cannot track all available BeiDou-3 signals. Because BeiDou-3 products are not available for most of the ACs, only BDS-2 constellation was taken into consideration in this study. Hereafter in this manuscript, BDS-2 will be referred to as BeiDou.

Some ACs produce the precise orbit and clock products of Galileo and BeiDou-2 constellations. Center for Orbit Determination in Europe (CODE) GeoForschungsZentrum (GFZ) and Wuhan University (WUHAN), are among these ACs. For time-constrained applications, ultra-rapid and rapid products from these ACs are also available. In this study, Galileo and BeiDou orbit accuracy was investigated using the rapid products from CODE, GFZ and WUHAN. Due to the high accuracy, rapid product is much more eligible for time-constrained applications that require high accuracy. High interest among the surveying community has been mainly focusing on the Galileo and BeiDou integration to GNSS. Some studies [7;8;9] related to rapid orbit accuracy using different products were investigated using Precise Point Positioning (PPP) and relative positioning techniques. But these studies were mainly conducted to evaluate GPS and GLONASS rapid orbit accuracy. Galileo and BeiDou orbit accuracy derived from the rapid products are still hot research topic. First, the processing strategy is presented in the following section. Then, the results are given and the work is concluded.

\section{Materials and Method}

Day of the year 1 through 31 in 2020 were chosen to investigate the Galileo and BeiDou orbit accuracy of rapid products derived by CODE, GFZ and WUHAN ACs. MGEX final product [10] was taken as a reference product for computing root mean square errors (RMSEs) of radial, alongtrack and cross-track components of the Galileo and BeiDou rapid products over the test period. CODE MGEX product provides the orbits for GPS, GLONASS, Galileo and BeiDou but excludes BeiDou geostationary (GEO) satellites due to the poor accuracy of GEO orbits [11]. Therefore, BeiDou Medium Earth Orbit (MEO) and Inclined Geo-Synchronous orbit (IGSO) satellites were evaluated. 15 min time interval was chosen for the orbit comparison for $24 \mathrm{~h}$ rapid and final products.

For the convenient interpretation of the relative location of the satellites between different orbits, local radial $\left(e_{R}\right)$, along-track $\left(e_{A}\right)$ and cross-track $\left(e_{C}\right)$ reference frame can be expressed as follows [2];

$e_{R}=\frac{r}{|r|}$

$e_{C}=\frac{r x \dot{r}}{|r x \dot{r}|}$

$e_{A}=e_{C} x e_{R}$ 
where $r$ and $\dot{r}$ are the satellite position and inertial velocity vector. RMSEs of radial, along-track and cross-track components of Galileo orbit were computed using CODE, GFZ and WUHAN rapid products while, RMSEs of BeiDou orbit components were computed using GFZ and WUHAN rapid products. BeiDou orbit does not included in the CODE rapid product for the test period.

\section{Results}

Figure 1 and 2 display the RMSEs of Galileo and BeiDou orbit using the rapid products from CODE, GFZ and WUHAN.

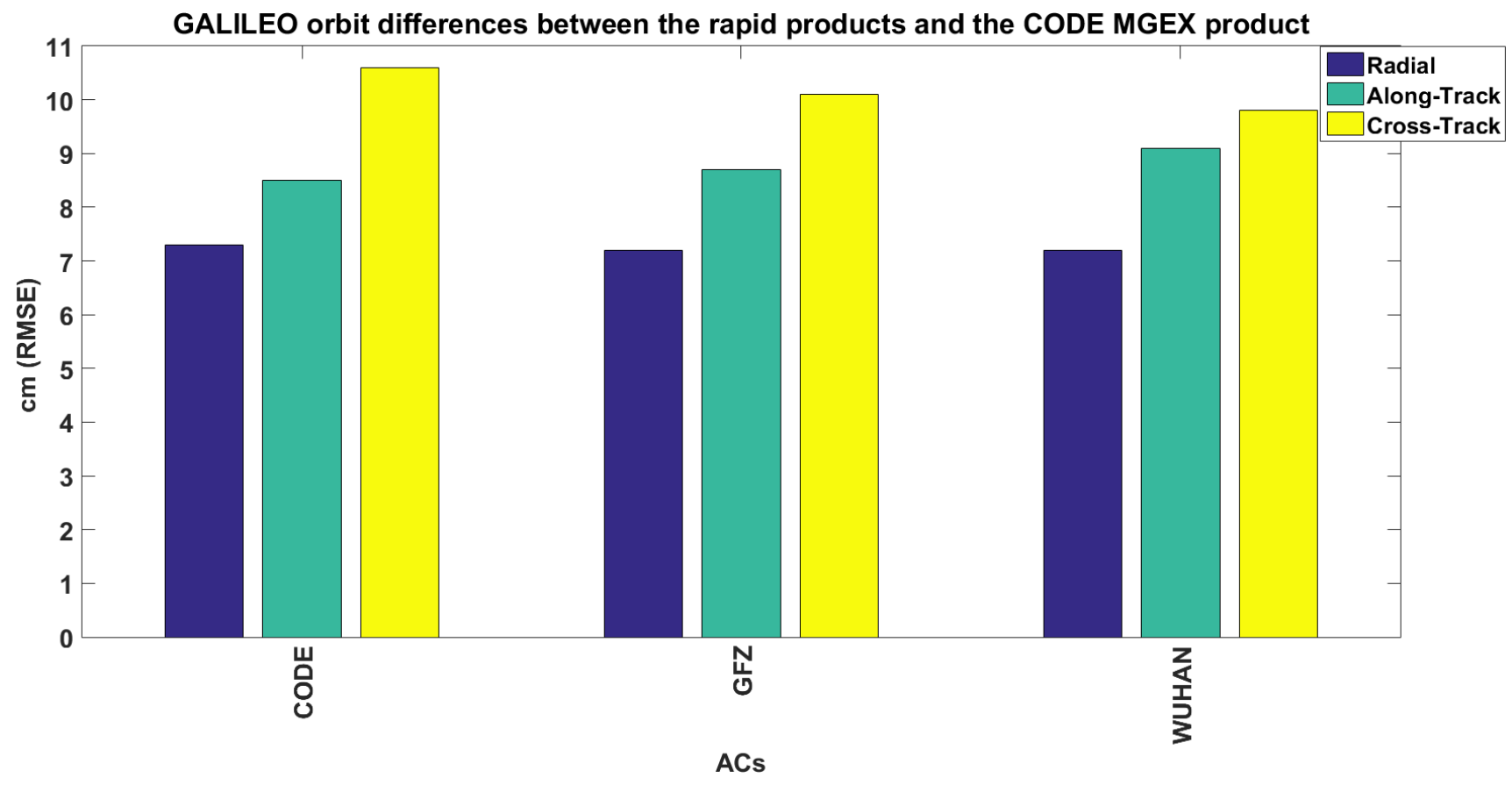

Figure 1. RMSEs of Galileo orbit 


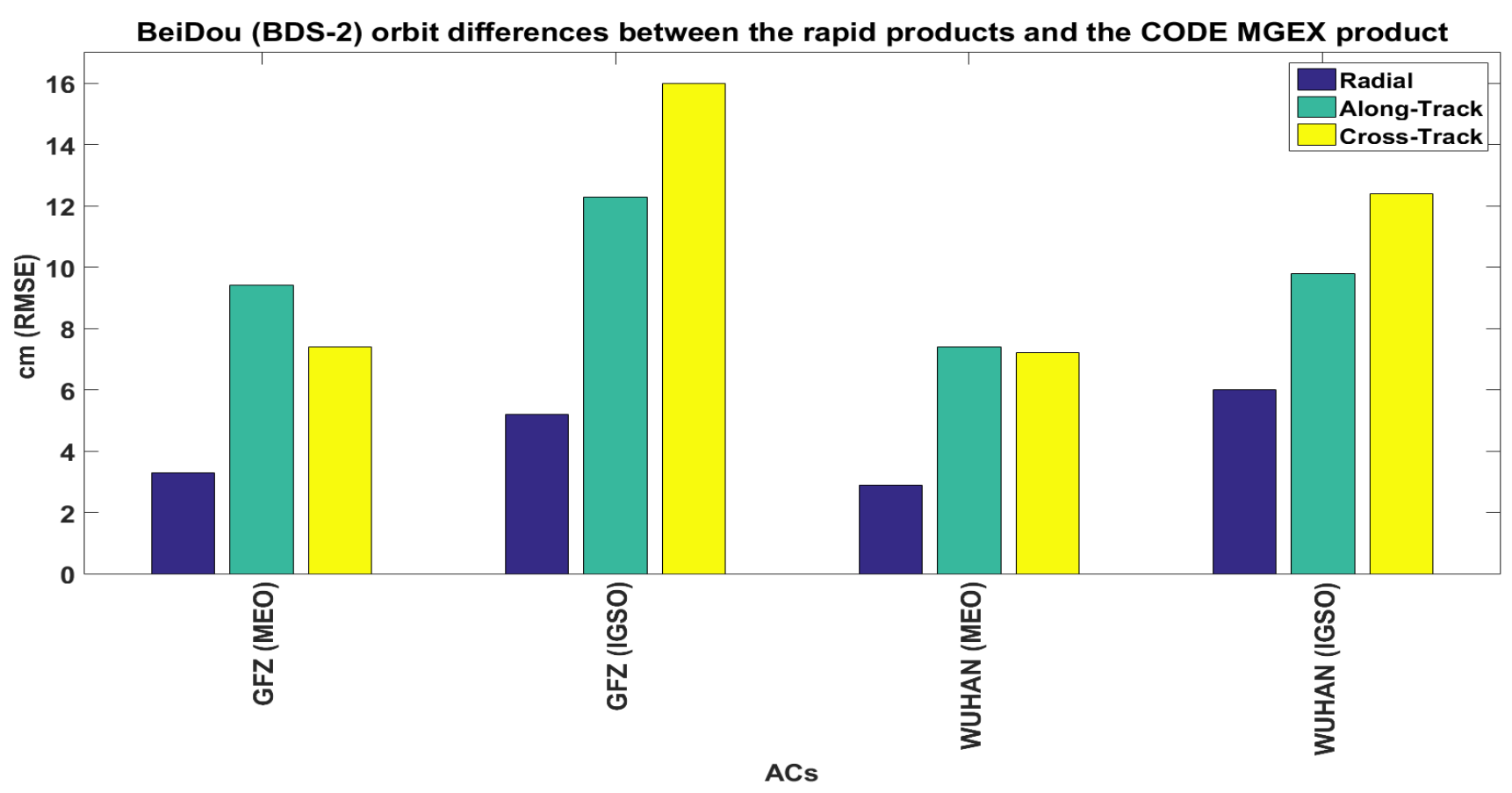

Figure 2. RMSEs of BeiDou orbit

As seen in the results, accuracy differences of Galileo orbit between the rapid products are not significant. Regarding the accuracy of BeiDou orbit, WUHAN rapid product produced the smaller RMSEs compared with the GFZ rapid product. The differences are much more evident in the alongtrack and cross-track components. The results also indicate that accuracy of along-track and crosstrack components are much lower compared with the radial component. Table 1 and 2 show the maximum absolute errors of Galileo and BeiDou orbit components, respectively, using the rapid products.

Table 1. Maximum absolute errors of Galileo

\begin{tabular}{|c|c|c|c|}
\hline \multirow{2}{*}{ Galileo } & \multicolumn{3}{|c|}{ Maximum Absolute Error (m) } \\
\hline & CODE & GFZ & WUHAN \\
\hline \multirow{3}{*}{$\begin{array}{c}\text { Radial } \\
\text { Along-Track } \\
\text { Cross-Track }\end{array}$} & 0.28 & 0.25 & 0.24 \\
\hline & 0.22 & 0.23 & 0.22 \\
\hline & 0.25 & 0.23 & 0.23 \\
\hline
\end{tabular}


Table 2. Maximum absolute errors of BeiDou

\begin{tabular}{ccccc}
\hline \multirow{2}{*}{ BeiDou } & \multicolumn{2}{c}{ Maximum Absolute Error $(\mathrm{m})$} \\
\cline { 2 - 5 } & \multicolumn{2}{c}{ GFZ } & \multicolumn{2}{c}{ WUHAN } \\
\cline { 2 - 5 } & MEO & IGSO & MEO & IGSO \\
\hline Radial & 0.05 & 0.09 & 0.04 & 0.10 \\
\cline { 2 - 5 } Along-Track & 0.10 & 0.20 & 0.11 & 0.18 \\
\cline { 2 - 5 } Cross-Track & 0.14 & 0.14 & 0.10 & 0.15 \\
\hline
\end{tabular}

As can be seen from the results of the maximum absolute errors, no significant maximum errors difference between the rapid products was observed. Maximum errors of radial component is significantly lower than the other components for BeiDou rapid orbit, whereas for Galileo rapid orbit, maximum errors of radial component is slightly higher than the other components.

\section{Conclusion}

For time-constrained applications, users need to choose the satellite orbit and clock products with minimum delay and maximum integrity. Rapid products are produced to mainly serve this issue. In this study, accuracy of Galileo and BeiDou orbits using the rapid products derived from CODE, GFZ and WUHAN ACs were investigated. Because GPS and GLONASS have reached the FOC, the orbit quality between the products are highly consistent. POD is much more important for the new emerging navigation systems such as Galileo and BeiDou, because FOC has not been declared yet except for BeiDou-2 regional system. Therefore, POD consistency among the ACs is not high as much as GPS and GLONASS and this can lead to the orbit accuracy differences. One month of data in 2020 was chosen to compute Galileo and BeiDou orbit accuracy using the rapid products. The results showed that marginal differences between the Galileo rapid products were found. Radial, along-track and cross-track accuracies were found approximately as $7 \mathrm{~cm}, 8.5 \mathrm{~cm}$ and 10 $\mathrm{cm}$ for the products. Accuracy differences between the BeiDou rapid products are rather high compared with the Galileo orbit. The smallest RMSEs were found using the WUHAN rapid products. As expected, the accuracy of the IGSO orbit is much lower compared with the MEO orbit for BeiDou constellation.

\section{References}

[1] Dow, J. M., Neilan, R. E., Rizos, C. (2009). The international GNSS service in a changing landscape of global navigation satellite systems. Journal of Geodesy, 83(3-4), 191-198, https://doi.org/10.1007/s00190-008-0300-3.

[2] Montenbruck, O., Steigenberger, P., Hauschild, A. (2015). Broadcast versus precise ephemerides: a multi-GNSS perspective. GPS Solutions, 19(2), 321-333, https://doi.org/10.1007/s10291-014-0390-8.

[3] Springer, T. A., and Hugentobler, U. (2001). IGS ultra rapid products for (near-) real-time applications. Physics and Chemistry of the Earth, Part A: Solid Earth and Geodesy, 26(6-8), 623-628, https://doi.org/10.1016/S1464-1895(01)00111-9. 
[4] http://www.igs.org/products Accessed: 16.07.2020

[5] Ogutcu, S. (2020). Performance assessment of IGS COMBINED/JPL individual rapid and ultra-rapid products: consideration of Precise Point Positioning technique. International Journal of Engineering and Geosciences, 5(1), 1-14, https://doi.org/10.26833/ijeg.577385.

[6] Montenbruck, O., Steigenberger, P., Khachikyan, R., Weber, G., Langley, R.B., Mervart, L., Hugentobler, U. (2014). IGS-MGEX: Preparing the Ground for Multi-Constellation GNSS Science. In Proceedings of the 4th International Colloquium on Scientific and Fundamental Aspects of the Galileo System, Prague, Czech Republic, 4-6 December 2013.

[7] Lu, F., and Li, J. (2011). Precise Point Positioning study to use different IGS precise ephemeris. In Computer Science and Automation Engineering (CSAE), 2011 IEEE International Conference, 3, 592-595, https://doi.org/10.1109/CSAE.2011.5952748.

[8] Park, J. K., and Jung, K. Y. (2014). Accuracy analysis of influences by satellite ephemeris. Contemporary Engineering Sciences, 7(24), 1, http://dx.doi.org/10.12988/ces.2014.49172.

[9] Martín, A., Anquela, A. B., Capilla, R., Berné, J. L. (2010). PPP technique analysis based on time convergence, repeatability, IGS products, different software processing, and GPS+ GLONASS constellation. Journal of Surveying Engineering, 137(3), 99-108, https://doi.org/10.1061/(ASCE)SU.1943-5428.0000047.

[10] Zhou, F., Cao, X., Ge, Y., Li, W. (2020). Assessment of the positioning performance and tropospheric delay retrieval with precise point positioning using products from different analysis centers. GPS Solutions, 24(1), 12, https://doi.org/10.1007/s10291-019-0925-0.

[11] Steigenberger, P., Hugentobler, U., Hauschild, A., Montenbruck, O. (2013). Orbit and clock analysis of Compass GEO and IGSO satellites. Journal of Geodesy, 87(6), 515-525, https://doi.org/10.1007/s00190-013-0625-4. 